\title{
Fine-needle aspiration cytology-efficacy in pre-surgical diagnosis of salivary gland tumors.
}

\author{
Paula López-Pazos $^{1}$, Mario Pérez-Sayáns ${ }^{1}$, Cintia Chamorro-Petronacci ${ }^{1 *}$, José M Suárez-Peñaranda ${ }^{2}$, \\ Marichalar-Mendia Xabier ${ }^{3}$, Mercedes Gallas Torreira ${ }^{1}$, Pilar Gándara-Vila ${ }^{1}$, Abel García García ${ }^{1}$ \\ ${ }^{1}$ Oral Medicine, Oral Surgery and Implantology Unit, Entrerríos s/n, Santiago de Compostela C.P., Spain \\ ${ }^{2}$ Department of Pathology and Forensic Sciences, University Hospital and School of Medicine of Santiago de \\ Compostela, Spain \\ ${ }^{3}$ Departamento de Estomatologia II, Universidad del Pais Vasco/Euskal Herriko Unibertsitatea, Barrio Sarriena, Leioa \\ Bizkaia, Spain
}

\begin{abstract}
The Fine-Needle Aspiration Cytology (FNAC) of salivary glands provides essential information in making clinical decisions. The purpose of this study is to assess the efficacy of FNAC in the pre-surgical diagnosis of salivary glands, comparing it to the final histopathological study. FNACs and biopsies collected from patients from $2000-2013$ period, were compared finding a total of 433 salivary gland lesions. Of the 433 salivary gland lesions in our study, FNAC was developed on just $22.8 \%$ of lesions, 96.9\% were satisfactory. 78 benign and 17 malignant lesions were diagnosed. In the histopathological study of lesions, 80 were diagnosed as benign and 18 as malignant. We found 14 true positive cases, 74 true negatives, 3 false positives and 4 false negatives. The diagnostic indexes included a FNAC sensitivity of $77 \%$ and a specificity of $96.1 \%$. A Positive Predictive Value (PPV) of $82.35 \%$ and a Negative Predictive Value (NPV) of $\mathbf{9 4 . 8 \%}$. For the diagnosis of salivary gland lesions, FNAC showed high sensitivity and specificity in our sample, and the level of agreement between the FNAC diagnosis and the final diagnosis is good.
\end{abstract}

Keywords: Fine-needle aspiration cytology, Salivary gland tumor, Diagnostic method.

Accepted on June 27, 2018

\section{Introduction}

The Fine-Needle Aspiration Cytology (FNAC) of salivary glands provides essential information in making clinical decisions for their appropriate treatment [1]. It is a simple technique that is easy to perform, cheap and rarely has complications [2-4].

Efficiency of FNAC is questioned by some authors and defended by another [3,5]. Layfield et al. [6] conducted a study on cost-effectiveness in regards to the FNAC of salivary glands and show that the fine-needle aspiration cytology can avoid the need for surgery by $35 \%$ of the masses diagnosed in the parotid gland.

FNAC surely distinguish between inflammatory lesions, which don't require a surgical procedure, and neoplastic lesions, differentiating between benign and malignant tumors, allowing the establishment of protocols of action on salivary gland tumors [7-9].
Low sensitivity and negative predictive value in screening tests can be attributed to the difficulty in diagnosis of low degree carcinomas, solely due to cell morphology [10].

Although FNAC is globally accepted as a pre-surgical technique in head and neck tumors, its effectiveness in neoplastic lesions of salivary glands is controversial due to the low sensitivity reported by some authors, who question its efficacy.

The purpose of this study is to assess the efficacy of FNAC in the pre-surgical diagnosis of salivary, parotid and submaxillary glands, comparing it to the final histopathological diagnosis of the surgical piece.

\section{Material and Methods}

\section{Samples}

Samples from the Pathological Anatomy Unit of the Santiago de Compostela Teaching Hospital were searched. Biopsies and FNACs from major and minor salivary glands had been collected during the $2000-2013$ period, which means a total of 
13 years of follow-up, finding samples from a total of 580 patients.

Exclusion criteria included: metastasis of primary tumors in other locations, studies in which the main lesion belonged to a tissue that didn't belong to the salivary gland due to the proximity of the sample to healthy gland tissue and, lastly, we unified the biopsies of the same lesion of the same patient but had been referenced as different anatomical and pathologic studies.

After exclusions, we had 433 studies from which we selected the biopsies of lesions of salivary glands, exclusively, since some of them presented several biopsy samples. In total, to develop the analysis of data we had a sample 433 biopsies of salivary glands which correspond to a study population of 433 patients, Caucasian, of which 179 were men and 254 were women $(41.3 \%$ men and $58.7 \%$ women), the gender ratio was 1.41:1 in favor of women, with ages comprehended between 3 and $97 \mathrm{y}$, and the average age was $51.29 \mathrm{y}$ (with a standard deviation of $17.34 \mathrm{y}$ ).

Variables analysed were: gender, ages of patients at the time of diagnosis of the lesion, development or not of the FNAC on the lesion, prior to the biopsy, cytologic diagnosis through FNAC, diagnosis of clinical suspicion, histologic variability of the tumor in its definitive diagnosis (WHO, WHO classification of salivary gland tumors 2005).

Considering these variables, we analysed sensitivity, specificity, positive predictive value, negative predictive value, false positive and false negative ratio of the test.

All procedures were carried out with the adequate understanding and written consent of the subjects.

This study has received the approval of ethical committee of University Hospital and School of Medicine of Santiago de Compostela.

\section{Results}

\section{Frequency of FNAC of salivary gland lesions}

FNAC was developed on $22.8 \%$ (98) of the total lesions (433). In all cases, biopsy was developed to confirm histology. FNAC wasn't developed on $332(77.2 \%)$ of patients and in 3 cases $(0.7 \%)$, we found no record of the development of FNAC or not (Figure 1).

\section{Cyto-histologic correlation in the diagnosis with FNAC}

$96.9 \%$ (95) of the total FNACs (98) were satisfactory and allowed to develop a cytologic diagnosis; the other 3 cases $(3.1 \%)$ were not diagnostic, the sample of these three lesions was insufficient to develop a correct diagnosis. 78 benign and 17 malignant lesions were diagnosed through cytology. In the histopathological study of lesions, 80 were diagnosed as benign and 18 as malignant.
We found 14 true positive cases (malignant cytology and histology), 74 true negatives (benign cytology and histology), 3 false positives (malignant cytology and benign histology) and 4 false negatives (benign cytology and malignant histology).

Of the 4 false negatives, two were diagnosed as pleomorph adenomas in the cytology, histologically, these resulted in a mucoepidermoid carcinoma and a NOS adenocarcinoma: another one was diagnosed as a Warthin tumor which was an ancinar cell carcinoma; and lastly a reactive lymphadenitis that turned out to be a Hodgkin lymphoma.

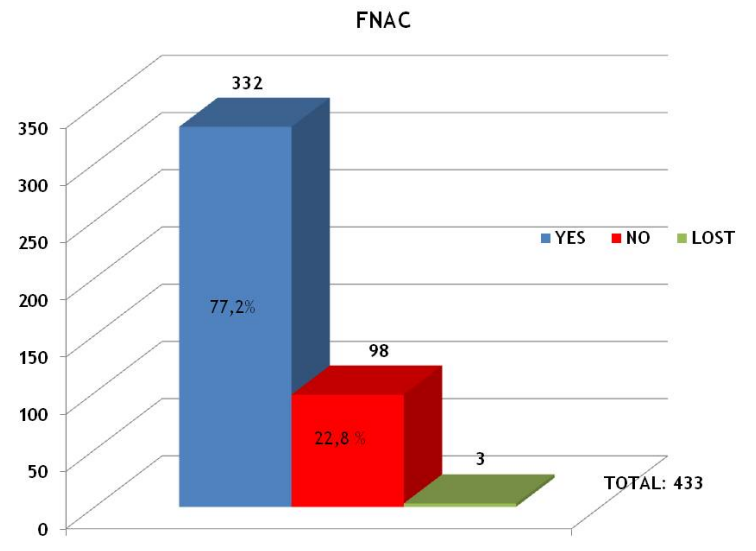

Figure 1. Frequency and proportion of the development of FNAC on tumoral and non-tumoral lesions under study.

Of the 3 false positives, two were lymphomas, which histologically were a non-pathological lesion and a sialadenitis; whereas the third case was an epidermoid carcinoma that turned out being a Warthin tumor.

Considering this data, the values of the diagnostic indexes included a FNAC sensitivity to detect malignancy in $77 \%$ and a specificity of $96.1 \%$. A Positive Predictive Value (PPV) of $82.35 \%$ and a Negative Predictive Value (NPV) of $94.8 \%$. The Rate of False Negatives (FNR) was 23\% and the Rate of False Positives (FPR) was 3.9\%.

Another data obtained in our result analysis was the degree of concordance or agreement between the diagnosis of clinical suspicion and the diagnosis through FNAC, and between the diagnosis by FNAC and the final diagnosis. For the former we obtained a minuscule concordance (Kappa index: 0.07), while for the latter, we obtained a good concordance level (Kappa index: 0.654).

\section{Discussion}

FNAC was only developed in the $22.8 \%$ of all cases due to the fact that the FNAC was not conducted when the practitioner strongly believed it was a tumor.

From the total lesions of our study (433), only 98, $22.8 \%$ underwent diagnosis by FNAC, the percentage of nondiagnostic FNACs is very low, just $2 \%$ in comparison to other studies in which FNAC is developed systematically in all 
lesions [11]. Therefore, the clinician must determine the need to develop a FNAC following the direct examination of the lesion and the development of additional tests, applying this protocol the number of non-diagnostic FNACs is reduced in most of the studies we reviewed [12].

In the current reviewed literature, sensitivity reached a range between $86.6 \%$ and $57.1 \%$, and specificity between $99 \%$ and 91\% [3,11-14] (Table 1). The meta-analysis developed by Schmidt et al. [15], in which they analysed a total of 6169 cases, shows a sensitivity and a specificity of $80 \%$ and $97 \%$, respectively, values that were very similar to those we obtained in our study (77\% sensitivity and $96 \%$ specificity). Our high sensitivity level is similar to those values found in the literature, except for one study that found values of $57.1 \%$. The authors justify these low values arguing that the FNACs and the anatomical and pathological studies were not developed by a sole operator. In our study, the FNACs were developed by maxillofacial surgeons from the Santiago de Compostela Teaching Hospital and, although it was always the same Pathological Anatomy Unit of the same hospital, the sampling wasn't developed by the same pathologist.

There are different reasons for these variables, it is very hard to compare data of different studies due to the methods developed in the classification of patients, their analysis and their results. In many cases, the studies analysed parotid gland lesions, however, in our study we included also cases from the submaxillary gland. Other studies included cases with clinical suspicion of malignancy, this inclusion criteria affected sensitivity, specificity and the concordance level; on the other hand, there also are studies that include the FNACs developed routinely on all tumoral lesions and the resulting percentage of malignant tumors was very low [11].

In regards to false positives, there are studies showing that the definitive diagnosis of the lesion was a Warthin tumor in most cases, since many times they present metaplastic changes in the epithelium [14]. In our sample, we only found one Warthin tumor among the three false positives. The other two cases corresponded to non-pathological or inflammatory processes.

Different series shows that the rate of false negatives amounts from $13 \%$ to $29 \%$ [16], we found a rate of $23 \%$. In general, malignant tumors of salivary glands can be hard to diagnose for the pathologist, tumors have a low incidence and there is a large/huge variety of different histologic patterns. It has been published that acinar cell carcinomas are usually erroneously interpreted as a benign lesions by cytology [12,17], as shown in different FNAC publications which were able to diagnose only $68 \%$ of this type of carcinomas [18]. In our study we solely determined one case of ancinar cell carcinomas among 4 false negatives, which were interpreted as a Warthin tumor in the cytology.

In our study the PPV was $82.35 \%$, implying that a FNAC reported as malignant was actually a benign lesion in $17.65 \%$ of cases. On the other hand, the NPV was $94.8 \%$. Reviewing the literature, we find studies with similar results that support our findings. Specifically, we must highlight the difference with the study by Zerpa et al. [14] with a PPV of $50 \%$, this unusually low value is justifiable since the number of malignant tumors in their series is below $8 \%$.

We found concordance values between histologic diagnosis by FNAC and the definitive diagnosis (Kappa index: 0.654) was similar to other studies, such as the one by Piccioni et al. [11] who found high concordance levels (Kappa index: 0.85).

In conclusion, for the diagnosis of salivary gland lesions, FNAC showed high sensitivity and specificity in our sample, and the level of agreement between the FNAC diagnosis and the final diagnosis is good. Therefore, it is a useful and simple tool to study these lesions, and serves as an orientation for diagnosis and the individual planning of each case.

Table 1. Comparison between results of this study and former studies.

\begin{tabular}{|c|c|c|c|c|c|}
\hline Study & No. cases & Sensitivity (\%) & Specificity (\%) & PPV & NPV \\
\hline Piccioni et al. [14] & 176 & 81 & 99 & 93 & 98 \\
\hline Zerpa et al. [17] & 93 & 57.1 & 95.1 & 50 & 96.3 \\
\hline Ali et al. [4] & 129 & 84 & 98 & 93 & 95 \\
\hline Arul et al. [16] & 146 & 86.6 & 94.6 & 88.3 & 94.6 \\
\hline Muñoz Palza et al. [15] & 40 & 71 & 91 & 62.5 & 93.7 \\
\hline López Pazos et al. (This study) & 95 & 77 & 96.1 & 82.35 & 94.8 \\
\hline
\end{tabular}

PPV: Positive Predictive Value; NPV: Negative Predictive Value.

\section{Acknowledgment}

We wish to thank our colleagues from the Pathological Anatomy Unit of the Santiago de Compostela Teaching Hospital and the University of Santiago de Compostela.

\section{References}

1. Bottles K, Miller TR, Cohen MB, Ljung BM. Fine needle aspiration biopsy. Has its time come? Am J Med 1986; 81: 525-531. 
2. Christensen RK, Bjorndal K, Godballe C, Krogdahl A. Value of fine-needle aspiration biopsy of salivary gland lesions. Head Neck 2010; 32: 104-108.

3. Ali NS, Akhtar S, Junaid M, Awan S, Aftab K. Diagnostic accuracy of fine needle aspiration cytology in parotid lesions. ISRN Surg 2011; 2011: 721525.

4. Bajaj Y, Singh S, Cozens N, Sharp J. Critical clinical appraisal of the role of ultrasound guided fine needle aspiration cytology in the management of parotid tumours. J Laryngol Otol 2005; 119: 289-292.

5. Mcguirt WF, Mccabe BF. Significance of node biopsy before definitive treatment of cervical metastatic carcinoma. Laryngoscope 1978; 88: 594-597.

6. Layfield LI, Gopez E, Hirschowitz S. Cost efficiency analysis for fine-needle aspiration in the workup of parotid and submandibular gland nodules. Diagn Cytopathol 2006; 34: 734-738.

7. Zurrida S, Alasio L, Tradati N, Bartoli C, Chiesa F, Pilotti S. Fine-needle aspiration of parotid masses. Cancer 1993; 72: 2306-2311.

8. Ashraf A, Shaikh AS, Kamal F, Sarfraz R, Bukhari MH. Diagnostic reliability of FNAC for salivary gland swellings: a comparative study. Diagn Cytopathol 2010; 38: 499-504.

9. Stewart CJ, MacKenzie K, McGarry GW, Mowat A. Fineneedle aspiration cytology of salivary gland: a review of 341 cases. Diagn Cytopathol 2000; 22: 139-146.

10. Song TH, Song JS, Sung CO. Accuracy of core needle biopsy versus fine needle aspiration cytology for diagnosing salivary gland tumors. J Pathol Transl Med 2015; 49: 136-143.

11. Piccioni LO, Fabiano B, Gemma M, Sarandria D, Bussi M. Fine-needle aspiration cytology in the diagnosis of parotid lesions. Acta Otorhinolaryngol Ital 2011; 31: 1-4.

12. Munoz-Palza C, Cordero-Jimenez A, Tenor-Serrano R, Garcia-Mata R, Contreras-Molina P, Garcia-Munoz I.
Cyto-histological correlation in parotid gland tumors. Acta Otorrinolaringol Esp 2010; 61: 184-188.

13. Arul P, Akshatha C, Masilamani S, Jonathan S. Diagnosis of salivary gland lesions by fine needle aspiration cytology and its histopathological correlation in a tertiary care center of Southern India. J Clin Diagn Res 2015; 9: $7-10$.

14. Zerpa-Zerpa V, Cuesta-Gonzales MT, Agostini-Porras G, Marcano-Acuna M, Estelles-Ferriol E, Dalmau-Galofre J. Diagnostic accuracy of fine needle aspiration cytology in parotid tumours. Acta Otorrinolaringol Esp 2014; 65: 157-161.

15. Schmidt RL, Hall BJ, Wilson AR, Layfield LJ. A systematic review and meta-analysis of the diagnostic accuracy of fine-needle aspiration cytology for parotid gland lesions. Am J Clin Pathol 2011; 136: 45-59.

16. Zbaren P, Nuyens M, Loosli H, Stauffer E. Diagnostic accuracy of fine-needle aspiration cytology and frozen section in primary parotid carcinoma. Cancer 2004; 100: 1876-1883.

17. Klijanienko J, Vielh P. Fine-needle sample of salivary gland lesions. V: cytology of 22 cases of acinic cell carcinoma with histologic correlation. Diagn Cytopathol 1997; 17: 347-352.

18. Nagel H, Laskawi R, Buter JJ, Schroder M, Chilla R, Droese M. Cytologic diagnosis of acinic-cell carcinoma of salivary glands. Diagn Cytopathol 1997; 16: 402-412.

\section{*Correspondence to}

Cintia Chamorro-Petronacci

Oral Medicine, Oral Surgery and Implantology Unit Instituto de Investigación Sanitaria de Santiago (IDIS)

Spain 